\title{
Al drug discovery booms in China
}

\author{
Chinese upstart companies and IT goliaths hope to turn country's artificial intelligence prowess into world-leading \\ drug innovation. Do they have an edge over Western players?
}

C

hinese researchers are abuzz about the potential of artificial intelligence (AI) in drug design and development. Just last month, three Chinese players raised hefty sums to fuel their drug discovery plans. Insilico Medicine, a Hong Kong-based company with half its global operations in Shanghai, raised \$255 million to push drug candidates discovered by its AI into clinical trials and tune its algorithms to find new targets. That followed $\$ 100$ million pocketed by Beijing StoneWise Technology in April and $\$ 319$ million that Shenzhen-based XtalPi added to its coffers last September.

IT goliaths like Tencent, Baidu and ByteDance have also been turning their formidable AI capacity to drug design. With an ambitious national strategy aimed at AI dominance, a massive talent pool of AI researchers, a plethora of contract research organizations (CROs) with which to partner, and evolving health data privacy protections, Chinese AI companies are poised to compete in the global race to make more drugs faster and cheaper. But even if a population of 1.4 billion can supply the wealth of data that will give Chinese AI companies a head start over Western counterparts, questions remain as to the quality of the preclinical and clinical data being used to train company algorithms.

"If you come here and see what is happening, it impresses you," says Alex Zhavoronkov, founder and CEO of Insilico Medicine. Kai-Fu Lee, CEO of Sinovation Ventures, a venture capital firm based in Beijing that invests in AI companies, agrees: "China does have a state-of-the-art ability to build drug discovery systems."

But Chinese AI drug hunters are latecomers. Over the past decade, companies like Atomwise, BenevolentAI (London), Exscientia (Oxford), Relay Therapeutics and Numerate were working out big deals with multinational pharmaceutical companies. Even the two most prominent AI drug design companies now associated with China-Insilico Medicine and XtalPi-were both established in 2014 in the United States. At the time, Chinese AI drug research was getting little traction. "When I first came back to China in 2015 and started talking with Chinese pharmaceutical companies, it was apparent that most of them were not interested in or were not ready for $\mathrm{AI}$ in drug R\&D," says XtalPi co-founder Ma Jian.

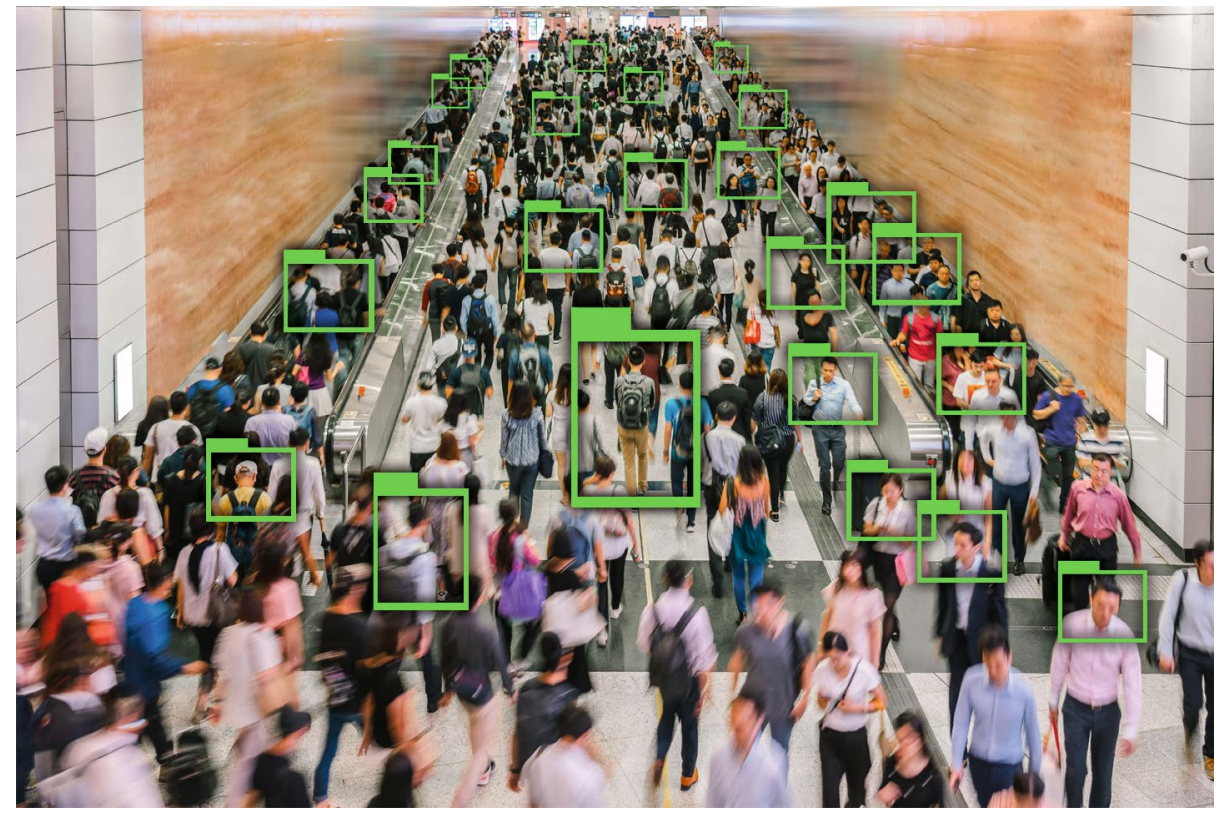

The Chinese government's regulatory reform is designed to boost Al-driven drug discovery, but attitudes toward data privacy continue to cause international concern. Credit: THANANIT SUNTIVIRIYANON / Alamy Stock Photo

Since then, though, the situation has changed rapidly. AlphaGo's victories over top human Go players in 2016 and 2017 focused industry attention on the potential of AI as a path to success in the minds of many young Chinese. The following year, the central government launched an ambitious framework to make China a global leader in AI by 2030 that led to, among other things, new university training programs.

\section{"Top Al experts in China cost more than in the United States and tend to move around."}

Around the same time, Chinese regulatory reform gave innovative drug discovery, including that based on AI, a boost. Updated bioequivalence rules in 2015 and new volume-purchasing stipulations have meant that innovative drugs are increasingly favored over generics, which had previously dominated China's drug market. The new rules tip the scales in favor of the quickest and most forward-looking manufacturers. That created a huge demand for new pipelines and with it a curiosity to see what AI had to offer. In 2019 Hansoh Pharmaceutical Group, for example, agreed to pay fees that could amount to over a billion dollars to Atomwise to design drugs targeting up to 11 proteins involved in cancer and other disorders. And while XtalPi has received most of it partnering money from US companies, China is now its fastest growing market. "For [Chinese] companies with little experience of innovation, AI represented a way to leapfrog," says Ma.

Researchers in China responded to the call. In a "storm of activity," computer scientists, physicists, mathematicians and traditional drug discovery researchers launched over 50 AI drug discovery companies, says Zhang Jian, who runs a molecular design laboratory at Shanghai Jiaotong University and has worked with a handful of AI drug design startups. In 2019, Zhang helped to reposition Shanghai-based Nutshell Therapeutics, which just raised its second installment of \$20 million in June, as an AI drug design company. In 2018, Zhou Jielong, a key force behind the evolution of Baidu's search engine, 
established StoneWise to aid in the discovery of small-molecule drugs.

Industry insiders and drug discovery researchers in the United States have remained skeptical of the AI hype and claims that AI can improve the attrition rate once drug programs reach human proof-of-concept studies. Chinese researchers argue that, given this conservative thinking, Chinese companies might be able to leverage the move to AI faster than Western companies: "China doesn't have a traditional way to do discovery, so there's no box. Those people don't have other rules in their mind," says $\mathrm{Yu}$ Zhengtian, co-founder of Nutshell, which uses AI to predict allosteric properties of disease targets.

China's large population and massive hospitals make it easy to aggregate large data sets-a crucial requirement for training AI. There is also less concern about privacy in China, meaning that the data are easier to access. Lee says that AI companies in the United States in particular are hobbled by regulations, such as the Health Insurance Portability and Accountability Act of 1996, which sets standards for how electronic health records can be shared. HIPAA makes it difficult, even when patients have given consent, to pull data together, says Lee. Although the Chinese government is about to come out with severe penalties for those who sell leaked personal data, companies like Yidu Cloud, which brings together consented patient data into a research tool, are emerging to ensure the data can be accessed, says Lee. Their counterparts in the US-Flatiron Health, Tempus, Aetion and Palantir-are similarly consolidating real-world electronic health record data on a massive scale for use in drug discovery.

But many of the data are of poor quality, which can mislead the AI. "Data quality is absolutely critical. Most Chinese companies working in drug discovery don't have good quality data for training," says Ye Tao, a medicinal chemist who uses computer-aided drug design at Peking University Shenzhen Graduate School.

Zhang agrees that data quality is a problem, especially outside of a few medical research hubs like Beijing and Shanghai. "It may be only possible to get good data in big cities. It's a little complex," he says.

China's formidable pool of AI researchers-comprising returnees from the United States and Europe; veterans of industrial research at Baidu, Alibaba and Tencent; and those being trained under new government programs supporting the country's long-term AI goals-should also give the country's sector a competitive edge. Among global AI research papers,
China-based researchers account for $22.4 \%$ of the total, compared with $16.4 \%$ from Europe and $14.6 \%$ from the United States, according to a recent report from Stanford University. And for the first time last year, AI publications from China garnered more citations than US ones. China still lacks scientists who will make fundamental breakthroughs, represented by the top $1 \%$ of the papers-especially the highly cited conference papers, says Lee. "When it comes to really the most creative out-of-the-box thinkers, the US still leads," he says.

But that might not matter for drug discovery. Chinese companies can tinker with AI algorithms based on generative adversarial networks or generative cooperative networks-most of which were developed in the United States, Canada or Europe-because they are often open source and publicly accessible. "You change a few words and change the whole concept. You can find a way to address the question you have," says Nutshell's Yu. "The science is from there [the West], but the technology is from here."

Lee says that the approach of Chinese AI drug discovery may also be more pragmatic, something he thinks gives China an edge. DeepMind, a Google subsidiary that has stunned researchers by solving a decades old protein folding riddle, might win the Nobel Prize or Turing award, but Chinese companies are "very good in monetizing AI, creating value with AI, building new products with AI," he says.

The explosion of interest and opportunities in AI has, however, driven up wages and made it difficult to retain expertise. Top AI experts in China cost more than in the United States and tend to move around, says Zhavoronkov. Insilico mainly hires AI expertise outside mainland China. Insilico, whose research stretches from a biological hypothesis and the identification of new biological targets to the production and testing of candidate molecules, mainly hires AI expertise outside mainland China and leans more heavily on Chinese research for later stage chemical design and molecule production, areas in which China excels. "We do geographic arbitrage," he says.

The bounty of CROs-some 3,000 altogether-in China is perhaps the most appealing aspect of China-based AI drug discovery firms, says Zhavoronkov. Insilico is partnering with 80 of them. This allows the company to avoid the loss of time and intellectual property that would happen if they worked with an academic institution. It also allows multiple experiments to be run in the development chain in parallel at multiple CROs. Comparing the results "is a

\section{WHO takes up CRISPR babies}

More than two years after the birth of twin girls with edited genomes, an advisory panel for the World Health Organization (WHO) has issued new recommendations that uphold the agency's initial reaction: that "it would be irresponsible to proceed with clinical applications of human germline genome editing." Such concerns over manipulation of human embryos are shared by the international scientific community, but few countries have regulations in place for the responsible use of genome editing techniques, and when they do have guidelines, they are often unenforceable. The WHO's 18-person expert panel has now come up with broad recommendations that address the scientific, ethical, social and legal implications raised by the prospect of editing the genome of a human embryo.

The first statement sets out five scenarios where gene editing might be applied-somatic genome editing for sickle cell disease, somatic gene editing for Huntington's disease, somatic gene editing by "unscrupulous" actors, somatic gene and epigenetic editing for enhancement, and germline editingand lays out the technical, ethical and societal issues that each faces. The second set of recommendations outlines the role that the WHO should undertake in establishing a moral and scientific leadership role. Among them are creating a registry of clinical trials involving gene editing and working with patent holders and regulatory agencies to insure that the technology, once developed, is widely accessible.

While these guidelines present a go-slow approach to gene editing, the opposite might be said about the International Society of Stem Cell Research (ISSCR) and its recommendation to relax the Warnock rule, which since 1984 has held that research on human embryos should not proceed beyond 14 days. The society is now calling for public discussions among stakeholders in different jurisdictions to decide whether experiments should be allowed to proceed beyond the 14 day limit.

Published online: 10 August 2021 https://doi.org/10.1038/s41587-021-01027-x 


\section{CRISPR super-sizes corn}

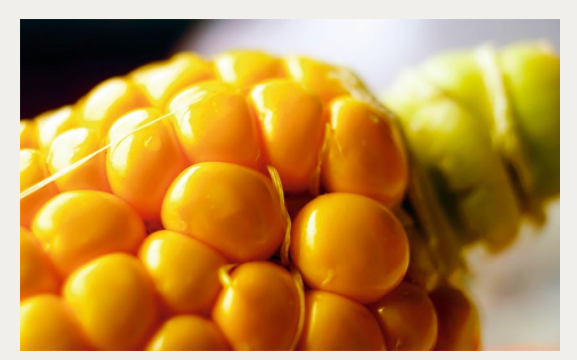

Gene-edited corn has more rows per ear. Credit: Kerem Arikan / Alamy Stock Photo

Biotech startup Pairwise Plants has launched field trials in the US Midwest, with backing from Bayer Crop Science, of corn with more kernel rows. The genetically edited corn, obtained with CRISPR base editing technology, has more than the average 16 rows on an ear, a trait, designed to help farmers increase efficiency and boost yields. Pairwise was founded by CEO Tom Adams and Chief Business Officer Haven Baker, with co-founders David Liu of Harvard University, Feng Zhang of the Massachusetts Institute of Technology and J. Keith Joung of Massachusetts General Hospital, to use CRISPR base editing technology to produce new varieties of fruit and vegetables with improved taste, longer shelf life, better yield, easier harvesting, and better adaptation to different weather conditions and season availability. In 2018, Pairwise raised \$25 million from investors and entered a \$100 million R\&D collaboration with Monsanto (now Bayer Crop Science) focused on corn, soybeans, wheat, cotton and canola. In February 2021, Pairwise raised $\$ 90$ million to develop its own first product: a salad leaf with the nutritional elements of kale but the eating qualities of green leaves that people find delicious, like romaine lettuce. Pairwise is also part of a public-private partnership to identify new traits in berries in the genus Rubus that allow low-labor picking and year round growth with maximum yield.

Because CRISPR-edited plants can reach the market without the US Department of Agriculture's oversight, Pairwise and its competitors, such as Caribou Biosciences and collaborator DuPont Pioneer, expect to avoid the hurdles faced by developers of traditional genetically modified crops.

Published online: 10 August 2021 https://doi.org/10.1038/s41587-021-01028-w huge learning experience for your AI," says Zhavoronkov.

"You've got enormous number of labs that can perform all experiments for you, at a reasonable cost, but at the same time in parallel," says Zhavoronkov. "The only place where you can do it is China."

So far Insilico and XtalPi, like their Western counterparts, have reached some preclinical milestones. Insilico uncovered a biological target linked to idiopathic pulmonary fibrosis and then designed a 'novel' molecule - at least, novel enough for an intellectual property claim-from scratch to match that target and potentially treat the disease. According to the company's press releases, the feat took less than 18 months and cost only about $\$ 2$ million. That's a fraction of what these stages typically cost. And according to a press release from XtalPi, its collaboration with Signet Therapeutics identified a gastric cancer treatment in six months. The company says it is now moving toward clinical trials. Shanghai Jiaotong University's Zhang says two compounds he has worked on are awaiting approval to begin clinical trials; he expects one of the trials to start next year. Nutshell's oncology candidate, meanwhile, is couple years away from clinical trials, Yu says.

But the success of AI drug design in China, as elsewhere, will depend on whether its candidates are successful in the clinic, something that no AI-designed drug has yet achieved. “There's a lot of hype, a lot of people try to sell you things that just aren't there," says Tudor Oprea, a computational biologist at the University of New Mexico. "The proof of the pudding is in the eating. So you don't really know until it's on the market."

Oprea says Insilico, for which he became scientific advisor, is a promising model because it takes on an "end-to-end" validation process, whereas many of the others look at a very small piece of the puzzle, making it difficult to know whether the AI is giving a meaningful result. "There's a lot of narrow-band vision," he says.
The entrance of Tencent, Baidu and ByteDance into AI drug discovery will add weight to China's efforts. In July 2020, Tencent launched its own AI-driven drug design platform, called iDrug. It already has already launched over ten projects, including a quest for drugs that work against coronaviruses. The company plans to cover the gamut of preclinical research, predicting protein structures, screening candidates, designing and optimizing molecules, and characterizing the functions of the proteins.

\section{"There's a lot of hype, a lot of people try to sell you things that just aren't there."}

Lee says these IT giants will act as China's version of Google, producing huge machines like DeepMind that can take on problems, like protein folding, that require more computational power than smaller startups can muster. "So now that we have a small, nimble companies that may be more pragmatic and faster to make money, and then big giants," says Lee. "So it's a good, good division of labor."

While there is some healthy competition between China and the United States, many researchers are more worried that current political tensions could compromise synergies that benefit both countries in this emergent arena. Most of the investment in Chinese AI drug design is from the US while a third of authors on influential AI conference papers were educated in China but are mostly working in the United States. "So I'm really hoping that healthcare or drug discovery are areas that don't become a part of this decoupling talk, because this research is all for the good of humanity," says Lee.

David Cyranoski

Kyoto, Japan

Published online: 3 August 2021

https://doi.org/10.1038/s41587-021-01016-0 